\title{
Changing trends in the incidence (1999-2011) and mortality (1983-2013) of cervical cancer in the Republic of Korea
}

\author{
Yoon Park*, Champadeng Vongdala*, Jeongseon Kim, Moran Ki \\ Department of Cancer Control and Policy, Graduate School of Cancer Science and Policy, National Cancer Center, Goyang, Korea
}

\begin{abstract}
Cervical cancer is a well-known preventable cancer worldwide. Many countries including Korea have pursued the positive endpoint of a reduction in mortality from cervical cancer. Our aim is to examine changing trends in cervical cancer incidence and mortality after the implementation of a national preventive effort in Korea. Cervical cancer incidence data from 1999 to 2011 and mortality data from 1983 to 2013 were collected from the Korean Statistical Information Service. Yearly age-standardized rates (ASR) per 100,000 were compared using two standards: the 2005 Korean population and the world standard population, based on Segi's world standard for incidence and the World Health Organization for mortality. In Korea, the age-standardized incidence of cervical cancer per 100,000 persons declined from 17.2 in 2000 to 11.8 in 2011. However, the group aged 25 to 29 showed a higher rate in 2011 (ASR, 6.5) than in 2000 (ASR, 3.6). The age-standardized mortality rate per 100,000 persons dropped from 2.81 in 2000 to 1.95 in 2013. In the worldwide comparison, the incidence rates remained close to the average incidence estimate of more developed regions (ASR, 9.9). The decreasing mortality trend in Korea approached the lower rate observed in Australia (ASR, 1.4) in 2010. Although the incidence rate of cervical cancer is continuously declining in Korea, it is still high relative to other countries. Moreover, incidence and mortality rates in females aged 30 years or under have recently increased. It is necessary to develop effective policy to reduce both incidence and mortality, particularly in younger age groups.
\end{abstract}

KEY WORDS: Uterine cervical neoplasms, Cervix uteri, Incidence, Mortality, Age groups, Korea

\section{INTRODUCTION}

Globally, cervical cancer is a preventable cancer [1]. It is causally associated with infection with the oncogenic human papillomavirus (HPV), particularly the two most common strains, HPV 16 and 18 [2-4]. The development of various preventive

\footnotetext{
Correspondence: Moran Ki

Department of Cancer Control and Policy, Graduate School of Cancer Science and Policy, National Cancer Center, 323 Ilsan-ro, Ilsandong-gu, Goyang 410-769, Korea

Tel: +82-31-920-2736, Fax: +82-50-4069-4908, E-mail: moranki@naver.com

*Yoon Park \& Champadeng Vongdala have contributed equally to this work as joint first authors.
}

Received: Dec 26, 2014, Accepted: May 29, 2015, Published: May 29, 2015 This article is available from: http://e-epih.org/ (C) 2015, Korean Society of Epidemiology

(C) This is an open-access article distributed under the terms of the Creative Commons Attribution License (http://creativecommons.org/licenses/by/3.0/), which permits unrestricted use, distribution, and reproduction in any medium, provided the original work is properly cited. strategies and clinical approaches has achieved a reduced incidence of cervical cancer, and hence mortality, in a number of developed countries [5]. Previous studies have shown that the introduction of a screening program plays a critical role in the control of cervical cancer [6].

In 1988, Korea introduced the practice of population-based cervical cancer screening using the Papanicolaou (Pap) smear, initially providing this service to selected people insured by the Korea National Health Insurance Program [4]. The National Cancer Screening Program was established in 1999 and has since provided free biennial Pap smear tests for females aged over 30 years. In 2000, the National Health Insurance Law mandated the Pap test as part of a nationwide screening program [7-9]. Several studies have reported positive outcomes related to cervical cancer screening that show a significantly lower incidence of invasive cervical cancer (International Classification of Diseases, 10th revision [ICD-10]: code C53) and carcinoma in situ (CIS; ICD-10: D06) among Korean females who were screened two or more times than among unscreened females [10]. In 
short, since around 2000, the implementation of a populationbased screening program has reduced the overall cervical cancer incidence and mortality rates in Korea. However, there may be different trends in age groups that could influence the incidence and mortality rates.

The aim of the present study was to examine changing trends in cervical cancer incidence and mortality in Korea. Here, we discuss patterns in both incidence and mortality with respect to yearly comparisons in specified age groups and to a worldwide comparison.

\section{MATERIALS AND METHODS}

Cervical cancer was classified according to the International Classification of Diseases for Oncology, 3rd edition [11], and converted according to the ICD-10 [12]. The code for invasive cervical cancer is C53, classified as malignant neoplasm of cervix uteri. Statistical data in this study only included cases of invasive cervical cancer. Incidence and mortality data were collected directly from the Korean Statistical Information Service [13] and indirectly from the Korea Ministry of Health and Welfare, the National Health Insurance Service, and the Korean Central Cancer Registry $[14,15]$. Incidence and mortality rates were compared by age from 1999 to 2011 and from 1983 to 2013 respectively. Both incidence and mortality data were gathered from the online database that showed selected datasets after choosing the type of cancer, required statistical unit, age, sex, and duration of time. Age-standardized rates calculated using the 2005 Korean population (ASR, K), were mainly used to compare differences in yearly rates. The reference population data were collected from the Korean Statistical Information Service [16]. To analyze the age-specific incidence trends from 2000 to 2010, ASRs from three different years $(2000,2005$, and 2010) were used.

For the worldwide comparison, age-standardized incidence rates in developed regions as classified by the GLOBOCAN project were collected as follows: all regions of Europe, Northern America, Australia, New Zealand, and Japan [17]. We selectively collected data from the list of countries in the databases of Cancer Incidence in Five Continents (CI-5) [18]: France (eight registries), United Kingdom (nine registries), United States (Surveillance, Epidemiology, and End Results, nine registries), Canada (three registries), Australia (six registries), New Zealand and Japan (three registries). Since the available data were limited until 2007, the estimated incidence data from the 2012 GLOBOCAN project were also collected [17]. Incidence rates in Korea (Table 1) were converted based on Segi's world standard population (ASR, W) $[19,20]$. When comparing global trends in mortality rates, age-specific data were only available from three of the developed countries mentioned above. We collected data directly from the New Zealand Cancer Registry [21], the Australasian Association of Cancer Registries [22], and the National Cancer Center in Japan [23]. For mortality, ASRs were determined using the World Health Organization (WHO) world standard population (ASR,W) [24].

\section{RESULTS}

\section{Incidence rates of cervical cancer}

In Korea, the incidence rates of cervical cancer have significantly declined since 2001 . Specifically, the incidence has declined from 4,443 cases in 1999 to 3,760 cases in 2011. Crude rates and ASRs per 100,000 decreased from 18.9 and 18.6, respectively, in 1999 to 15.0 and 11.8 in 2011 (Table 1). Compared to the year 2000, a lower incidence was shown in all age groups in 2011 except in those aged under 30. Individuals aged 25 to 29 showed a higher incidence of 6.5 in 2011 compared to 3.6 in 2000 (Figure 1) [25].

In Figure 2, each birth cohort is represented at three points, depicting the incidence in 2000, 2005, and 2010. The birth cohort of 1976 to 1980 and the cohort of 1966 to 1970 showed similar incidences at ages 30 to 34 of 13.3 and 13.2 respectively. Older age groups showed higher incidences if they came from earlier birth cohort groups. Specifically, between the ages of 40 and 44, there was a higher incidence of 33.0 in the 1955 to 1960 birth cohort compared to that of 23.0 in the 1966 to 1970 cohort. Likewise, the rate of 35.5 in the 1945 to 1950 cohort was higher than that of 25.4 in the 1955 to 1960 cohort for individuals aged 50 to 54 , and the rate of 42.4 in the 1935 to 1940 cohort was higher than that of 26.9 in the 1945 to 1950 cohort for individuals aged 60 to 64 .

For the worldwide comparison, converted rates using Segi's world population appear lower than the rates using the 2005 Korean population, such that incidence changes from 18.6 per 100,000 (ASR, K) to 16.3 per 100,000 (ASR, W) in 1999 and from 11.7 per 100,000 (ASR, K) to 10.1 per 100,000 (ASR,W) in 2011. Compared to more developed countries, incidence rates in Korea are relatively high (Figure 3). For instance, the ASR of 12.4 per 100,000 in 2005 is similar to that of New Zealand in 1990 (ASR, W: 12.65 per 100,000), which was the highest reported rate in 1990 among comparable countries. From the 1990s to 2007 , all other countries compared showed lower incidence than Korea, and showed a steadily decreasing pattern during this time. The GLOBOCAN project estimated the cervical cancer incidence rates in 2012. Due to the different regional classifications used in the GLOBOCAN and CI-5, only two among eight countries could be compared in this study (Figure 3): an ASR of 9.5 per 100,000 in Korea and 5.3 per 100,000 in New Zealand. 
Table 1. Incidence rate (1999-2011) and mortality rate (1983-2013) by year of cervical cancer in Korea

\begin{tabular}{|c|c|c|c|c|c|c|}
\hline \multirow[b]{2}{*}{ Year } & \multicolumn{3}{|c|}{ Incidence } & \multicolumn{3}{|c|}{ Mortality } \\
\hline & Cases & $\begin{array}{c}\text { Crude rate } \\
\text { (per 100.000) }\end{array}$ & $\begin{array}{c}\text { ASR }^{1} \\
(\text { per 100.000) }\end{array}$ & Cases & $\begin{array}{c}\text { Crude rate } \\
\text { (per 100.000) }\end{array}$ & $\begin{array}{c}\text { ASR }^{1} \\
\text { (per 100.000) } \\
\end{array}$ \\
\hline 1983 & - & - & - & 129 & 0.7 & 0.98 \\
\hline 1984 & - & - & - & 147 & 0.7 & 1.09 \\
\hline 1985 & - & - & - & 177 & 0.9 & 1.26 \\
\hline 1986 & - & - & - & 189 & 0.9 & 1.26 \\
\hline 1987 & - & - & - & 222 & 1.1 & 1.49 \\
\hline 1988 & - & - & - & 265 & 1.3 & 1.67 \\
\hline 1989 & - & - & - & 359 & 1.7 & 2.21 \\
\hline 1990 & - & - & - & 343 & 1.6 & 2.07 \\
\hline 1991 & - & - & - & 360 & 1.7 & 2.11 \\
\hline 1992 & - & - & - & 439 & 2.0 & 2.38 \\
\hline 1993 & - & - & - & 425 & 1.9 & $2.25^{2}$ \\
\hline 1994 & - & - & - & 560 & 2.5 & $2.91^{2}$ \\
\hline 1995 & - & - & - & 554 & 2.4 & $2.76^{2}$ \\
\hline 1996 & - & - & - & 668 & 2.9 & $3.28^{2}$ \\
\hline 1997 & - & - & - & 680 & 2.9 & $3.11^{2}$ \\
\hline 1998 & - & - & - & 610 & 2.6 & $2.56^{2}$ \\
\hline 1999 & 4,443 & 18.9 & 18.6 & 690 & 2.9 & $2.87^{2}$ \\
\hline 2000 & 4,253 & 18.0 & 17.2 & 726 & 3.1 & $2.81^{2}$ \\
\hline 2001 & 4,572 & 19.2 & 18.0 & 807 & 3.4 & $2.84^{2}$ \\
\hline 2002 & 4,402 & 18.4 & 16.8 & 1,009 & 4.2 & $3.42^{2}$ \\
\hline 2003 & 4,373 & 18.2 & 16.2 & 1,111 & 4.6 & 3.60 \\
\hline 2004 & 4,130 & 17.1 & 14.9 & 1,078 & 4.5 & 3.28 \\
\hline 2005 & 4,014 & 16.5 & 14.1 & 1,066 & 4.4 & 3.05 \\
\hline 2006 & 4,047 & 16.6 & 13.9 & 1,002 & 4.1 & 2.78 \\
\hline 2007 & 3,755 & 15.3 & 12.7 & 987 & 4.0 & 2.72 \\
\hline 2008 & 4,004 & 16.2 & 13.2 & 954 & 3.9 & 2.48 \\
\hline 2009 & 3,803 & 15.3 & 12.2 & 950 & 3.8 & 2.32 \\
\hline 2010 & 3,956 & 15.9 & 12.6 & 956 & 3.8 & 2.21 \\
\hline 2011 & 3,760 & 15.0 & 11.8 & 989 & 4.0 & 2.31 \\
\hline 2012 & - & - & - & 889 & 3.5 & 2.06 \\
\hline 2013 & - & - & - & 892 & 3.5 & 1.95 \\
\hline
\end{tabular}

Source from Korean Statistical Information Service. Death causes by 5-year age group and gender, mortality rates (1983-2013); 2014 [13]; Korean Statistical Information Service. Number of cancer patients, relative frequency, crude rate, age-adjusted incidence by cancer site and sex from 1999 to $2011 ; 2014$ [25] ASR, age-standardized rates.

${ }^{1}$ ASR per 100,000 using the Korea population of 2005.

${ }^{2}$ Corrected mortality using national death certification data. ASR using direct method based on 1967 World Health Organization world standard population (from 1993 to 2002 by year: 5.20, 5.60, 5.10, 5.10, 4.90, 4.30, 4.20, 4.10, 4.00, 3.90, respectively). Adapted from Shin HR, et al. Int J Cancer 2008;122:393397 [4].

\section{Mortality rates of cervical cancer}

In Korea, the annual mortality increased from 129 in 1983 to 892 in 2013. Crude rates and ASRs per 100,000 changed from 0.7 and 0.98 , respectively, in 1983 to 3.5 and 1.95 in 2013 (Table 1). However, when interpreting these mortality data, it should be understood that a limitation of this study is the use of uncorrected mortality records from death certificates that misclassified cervical cancer in Korea. A study conducted in 2007 corrected the number of cervical cancer deaths by comparing death certificates from cases containing data on unspecified uterine cancer with the national cancer incidence database of the entire cancer registry in Korea. After the correction, there was an ap- parent reduction in mortality; the overall age-standardized mortality rate per 100,000 persons decreased from 5.2 in 1993 to 3.9 in 2002, based on the $1967 \mathrm{WHO}$ world standard population [4]. From 2003 to 2013, there was a gradual reduction of cervical cancer mortality in Korean females.

Groups aged 50 and over generally had a higher proportion of overall mortality from cervical cancer. From the ages of the mid-30s to mid-40s, the mortality rates increased significantly. During the years from 2000 to 2011, mortality rates were not changed or improved in any age group except in the older groups aged in the mid-50s and above. In certain age groups, mortality rates were higher in the more recent year of 2011 than in 2000. 


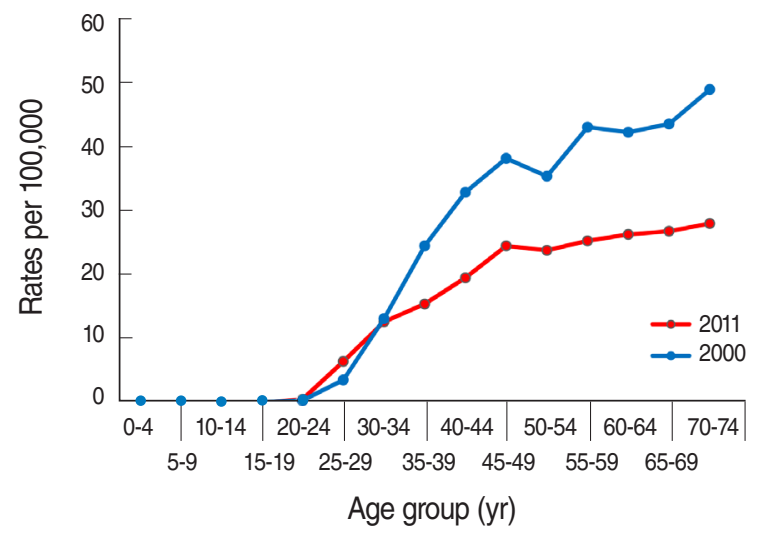

Figure 1. Age-specific incidence rates per 100,000 populations of cervical cancer in Korea, 2000 and 2011. Source from Korean Statistical Information Service. Number of cancer patients, relative frequency, crude rate, age-adjusted incidence by cancer site and sex from 1999 to 2011; 2014 [25].

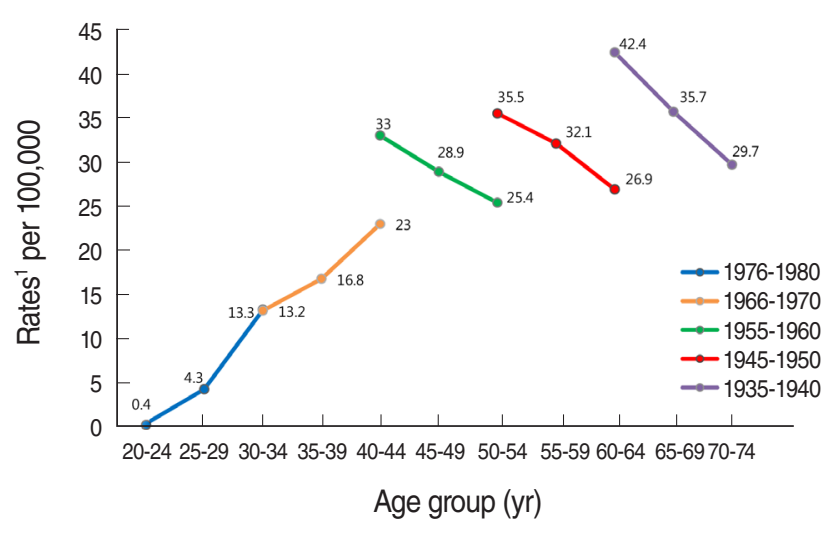

Figure 2. Trends in age-specific incidence rates of cervical cancer by birth cohort group in Korea, 2000, 2005 and 2010. Source from Korean Statistical Information Service. Number of cancer patients, relative frequency, crude rate, age-adjusted incidence by cancer site and sex from 1999 to 2011; 2014 [25]. ${ }^{1}$ Age-adjusted to the 2005 Korea standard population.

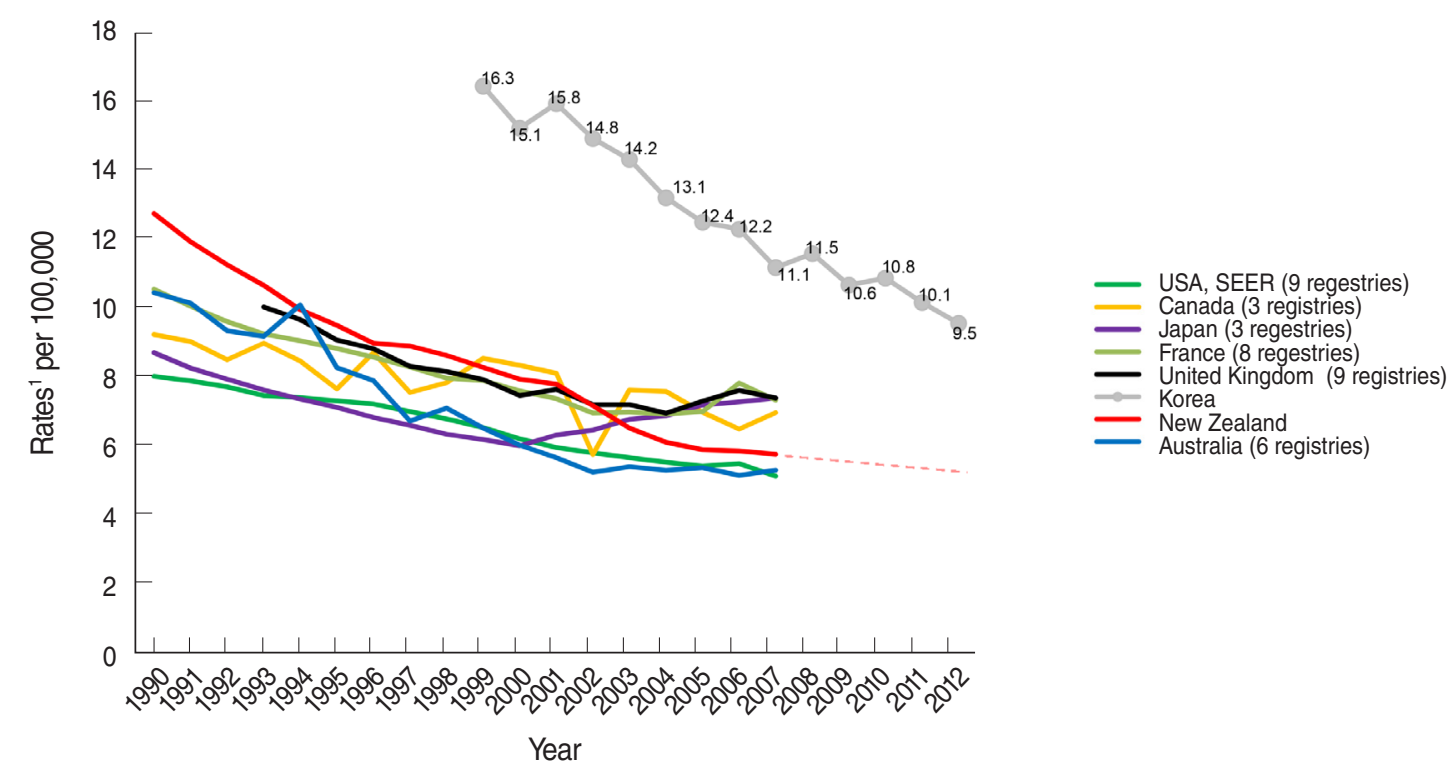

Figure 3. Trends of incidence rates of cervical cancer, worldwide, 1990-2012². Source from International Agency for Research on Cancer. GLOBOCAN 2012: estimated cancer incidence, mortality and prevalence worldwide in 2012; 2014 [17]; International Agency for Research on Cancer. Cancer incidence in five continents volume X(Cl5X); 2013; 2014 [18]. SEER, Surveillance, Epidemiology, and End Results. ${ }^{1}$ Ageadjusted to Segi 's world standard population; ASR, age-standardized rates. ${ }^{2} E$ stimated incidence rate in ASR (W), 2012: the estimates incidence rates in the year of 2012 are representable for each country as a whole while the data from $\mathrm{Cl}-5$ were collected selectively by cancer registry regions.

The age groups of 30 to 34 and 50 to 54 showed significantly higher mortality rates in 2011, with 0.13 per 100,000 and 0.36 per 100,000 , respectively, compared to 0.06 per 100,000 and 0.30 per 100,000 in 2000 (Figure 4).

For the worldwide comparison, three countries including Korea showed a decreasing pattern over the years studied (Figure 5). For instance, the ASR in Australia of 3.1 per 100,000 in 1991 was similar to that of Korea in 2003 and of New Zealand in 2001 (ASR, W: 3.09 per 100,000 and 3.0 per 100,000, respectively); these different years reflect a trend in each country of decreasing mortality rates. Notably, although the mortality rates showed a gradual decline in Korea, the mortality rate in 2013 (ASR,W: 1.65 per 100,000) was still higher than that of Australia in 2010 (ASR, W: 1.4 per 100,000). 


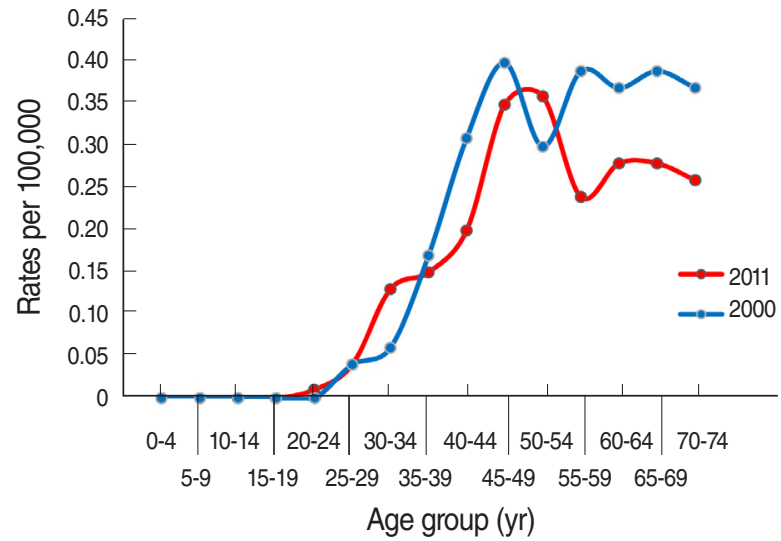

Figure 4. Age-specific mortality rates per 100,000 populations of cervical cancer in Korea, 2000 and 2011. Source from Korean Statistical Information Service. Death causes by 5 -year age group and gender, mortality rates (1983-2013); 2014 [13].

Since 2010, a pattern of slightly increasing mortality has been observed in Japan, which first reported a higher mortality rate (ASR, W: 1.97 per 100,000) than that of Korea (ASR, W: 1.88 per 100,000) in 2010.

\section{DISCUSSION}

Both incidence and mortality rates of cervical cancer in Korea have been declining since the nationwide cancer screening program was implemented [10]. Despite a decrease in cervical cancer cases, the age-standardized incidence standardized against the Korean population is higher than the age-standardized incidence using the world standard population. This is due to the relatively higher distribution of the elderly in Korea compared with the demographics of the world population.

In Korea, invasive cervical cancer appears to have increased predominantly among the younger age group [26]. The increasing incidence in individuals aged 20 to 29 and relatively slight reduction in mortality in all age groups might be the result of cases with more advanced cancer at diagnosis and consequent poor prognosis and low survival rates [27,28]. Since cure rates depend strongly on the stage at diagnosis [29], detection at an advanced stage might be closely related to unchanged mortality rates over time. At this late stage, cancer detection might occur at voluntary hospital visits after observation of relevant signs or symptoms [30] rather than by regular screening tests.

The significantly high mortality in groups aged in the late 30 s, late 40s, and above may reflect potential risk factors. Early initiation of sexual activity might cause early exposure to HPV infection [31]. Females infected with an oncogenic HPV type will progress to high-grade cervical intraepithelial neoplasia in their

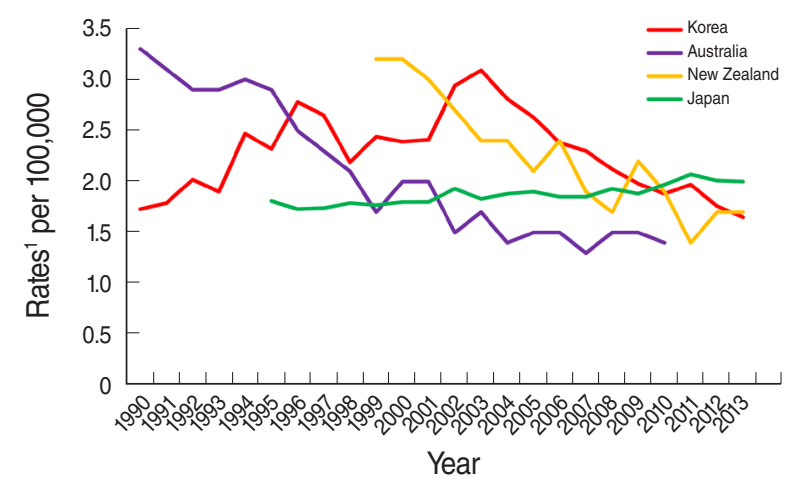

Figure 5. Trends of mortality rates of cervical cancer, worldwide, 1990-2013. Source from Korean Statistical Information Service. Death causes by 5-year age group and gender, mortality rates 1983-2013; 2014 [13]; Ministry of Health. Cancer: historical summary 1948-2011; 2014 [21]; Australian Institute of Health and Welfare. Australian cancer incidence and mortality (ACIM) book for cervical cancer; 2014 [22]; Japan National Cancer Center. Cancer mortality from vital statistic in Japan 1958-2013; 2014 [23]. 'Ageadjusted to World Health Organization world standard population.

20s or early 30s. The latency from HPV infection to cancer development is long, such that invasive cancer may appear 10 or more years after infection [29]. Moreover, elderly females might receive more exposure to low hygiene environments that increase the risk of HPV infection [32] and have less opportunity to engage with preventive programs from a young age. Despite the introduction of HPV vaccination, the current vaccines do not cover all potential oncogenic HPV types [29] and vaccination does not prevent cancer among individuals already infected with HPV.

In countries where organized cytological screening has been implemented over the last three or four decades, cervical cancer incidence and mortality rates have declined steadily [33, 34]. In the US, for example, incidence has been falling at a consistent rate across most states, declining by $1.9 \%$ per year. Overall mortality from cervical cancer has also been falling at a consistent rate, and this pattern in mortality is similar to that of Korea at present [35]. To obtain a reduction in mortality, the US has provided free or low-cost cervical cancer screening and health services for more than 20 years. Moreover, it has made a continuous effort to increase the proportion of cervical cancer screening from $83 \%$ of the female population in 2010 to the Healthy People 2020 target of $93 \%$ [36]. It has also emphasized that nonfinancial barriers, such as lack of awareness of disease, need to be addressed to save more lives [37].

Exploring changing trends in cervical cancer raises an important question about the effectiveness of cervical cancer screening. Routine screening might not be an efficient sole means of protecting against cervical cancer, especially in the younger age 
group of 20 to 40 years in Korea. A combination of public awareness of causal factors, early cancer detection by regular screening with the incorporation of HPV DNA testing, and vaccination at reasonable costs will constitute key preventive tools against cervical cancer [38] in Korea, as well as on a global scale.

\section{CONFLICT OF INTEREST}

The authors have no conflicts of interest with the material presented in this paper.

\section{REFERENCES}

1. Whynes DK, Philips Z, Avis M. Why do women participate in the English cervical cancer screening programme? J Health Econ 2007; 26:306-325.

2. Cho E, Kang MH, Choi KS, Suh M, Jun JK, Park EC. Cost-effectiveness of Korea's National Cervical Cancer Screening Program. Asian Pac J Cancer Prev 2013;14:4329-4334.

3. Vaccarella S, Lortet-Tieulent J, Plummer M, Franceschi S, Bray F. Worldwide trends in cervical cancer incidence: impact of screening against changes in disease risk factors. Eur J Cancer 2013;49:32623273.

4. Shin HR, Park S, Hwang SY, Kim JE, Jung KW, Won YJ, et al. Trends in cervical cancer mortality in Korea 1993-2002: corrected mortality using national death certification data and national cancer incidence data. Int J Cancer 2008;122:393-397.

5. Schiffman M, Wentzensen N. Human papillomavirus infection and the multistage carcinogenesis of cervical cancer. Cancer Epidemiol Biomarkers Prev 2013;22:553-560.

6. Dehler S, Tonev S, Korol D, Rohrmann S, Dimitrova N. Recent trends in cancer incidence: impact of risk factors, diagnostic activities and data quality of registration. Tumori 2014;100:399-405.

7. Cho E, Kang MH, Choi KS, Suh M, Jun JK, Park EC. Cost-effectiveness outcomes of the national gastric cancer screening program in South Korea. Asian Pac J Cancer Prev 2013;14:2533-2540.

8. Sung NY, Park EC, Shin HR, Choi KS. Participation rate and related socio-demographic factors in the national cancer screening program. J Prev Med Public Health 2005;38:93-100 (Korean).

9. Kwak MS, Park EC, Bang JY, Sung NY, Lee JY, Choi KS. Factors associated with cancer screening participation, Korea. J Prev Med Public Health 2005;38:473-481 (Korean).

10. Jun JK, Choi KS, Jung KW, Lee HY, Gapstur SM, Park EC, et al. Effectiveness of an organized cervical cancer screening program in Korea: results from a cohort study. Int J Cancer 2009;124:188-193.

11. World Health Organization. International classification of diseases for oncology, 3rd ed (ICD-O-3); 2000 [cited 2014 Oct 26]. Available from: http://codes.iarc.fr/codegroup/1.

12. World Health Organization. International classification of diseases (ICD) [cited 2014 Oct 28]. Available from: http://apps.who.int/classifications/icd10/browse/2015/en\#/C51-C58.

13. Korean Statistical Information Service. Death causes by 5 -year age group and gender, mortality rates (1983-2013); 2014 [cited 2014 Oct 28]. Available from: http://kosis.kr/wnsearch/totalSearch.jsp (Korean). 14. Korea Central Cancer Registry. Cancer registry system in Korea; 2014 [cited 2014 Oct 26]. Available from: http://ncc.re.kr/cancerStatsList. ncc?searchKey=total\&searchValue $=$ \&pageNum=1.

15. Jung KW, Won YJ, Kong HJ, Oh CM, Lee DH, Lee JS. Cancer statistics in Korea: incidence, mortality, survival, and prevalence in 2011. Cancer Res Treat 2014;46:109-123.

16. Korean Statistical Information Service. Reference population of Korea (1992-2013); 2014 [cited 2014 Oct 28]. Available from:http://kosis.kr/wnsearch/totalSearch.jsp (Korean).

17. International Agency for Research on Cancer. GLOBOCAN 2012: estimated cancer incidence, mortality and prevalence worldwide in 2012 [cited 2014 Oct 20]. Available from: http://globocan.iarc.fr/Default.aspx.

18. International Agency for Research on Cancer. Cancer incidence in five continents volume X (CI5X); 2013 [cited 2014 Oct 20]. Available from: http://ci5.iarc.fr/CI5-X/Default.aspx.

19. International Agency for Research on Cancer. Glossary of terms from cancer incidence in five continents volume X (CI5X); 2013 [cited 2014 Oct 20]. Available from: http://ci5.iarc.fr/CI5plus/Pages/glossary.aspx.

20. Doll R. Cancer in five continents. Proc R Soc Med 1972;65:49-55.

21. Ministry of Health. Cancer: historical summary 1948-2011; 2014 [cited 014 Dec 8]. Available from: http://www.health.govt.nz/publication/cancer-historical-summary-1948-2011.

22. Australian Institute of Health and Welfare. Australian cancer incidence and mortality (ACIM) book for cervical cancer; 2014 [cited 2014 Dec 8]. Available from: http://www.aihw.gov.au/acim-books.

23. Japan National Cancer Cener. Cancer mortality from vital statistics in Japan (1958-2013); 2014 [cited 2014 Dec 8]. Available from: http: //ganjoho.jp/en.professional/ststistics/table_download.html (Japanese).

24. Ahmad OB, Boschi-Pinto C, Lopez AD, Murray CJL, Lozano R, Inoue M. Age standardization of rates: a new WHO standard; 2001 [cited 2014 Dec 8]. Available from: http://www.who.int/healthinfo/paper31.pdf.

25. Korean Statistical Information Service. Number of cancer patients, relative frequency, crude rate, age-adjusted incidence by cancer site and sex from 1999 to 2011; 2014 [cited 2014 Oct 28]. Available from: http://kosis.kr/statHtml/statHtml.do?orgId=117\&tblId=DT_117N_ A $00025 \&$ conn path $=$ I2 (Korean).

26. Oh CM, Jung KW, Won YJ, Shin A, Kong HJ, Jun JK, et al. Trends in the incidence of in situ and invasive cervical cancer by age group and histological type in Korea from 1993 to 2009. PLoS One 2013; 8:e72012.

27. Darlin L, Borgfeldt C, Widén E, Kannisto P. Elderly women above screening age diagnosed with cervical cancer have a worse prognosis. Anticancer Res 2014;34:5147-5151.

28. Nartthanarung A, Thanapprapasr K, Udomsubpayakul U, Thanapprapasr D. Age and survival of cervical cancer patients with bone metastasis. Asian Pac J Cancer Prev 2014;15:8401-8404.

29. Adami HO, Hunter DJ, Trichopoulos D. Textbook of cancer epidemiology. New York: Oxford University Press; 2008, p. 446-467.

30. Jung M. National cancer screening programs and evidence-based healthcare policy in South Korea. Health Policy 2015;119:26-32.

31. Beral V. Cancer of the cervix: a sexually transmitted infection? Lancet 1974;1:1037-1040.

32. McGraw SL, Ferrante JM. Update on prevention and screening of cervical cancer. World J Clin Oncol 2014;5:744-752.

33. Pontén J, Adami HO, Bergström R, Dillner J, Friberg LG, Gustafsson L, et al. Strategies for global control of cervical cancer. Int J Cancer 1995;60:1-26.

34. Gustafsson L, Pontén J, Zack M, Adami HO. International incidence rates of invasive cervical cancer after introduction of cytological screen- 
ing. Cancer Causes Control 1997;8:755-763.

35. Benard VB, Thomas CC, King J, Massetti GM, Doria-Rose VP, Saraiya $\mathrm{M}$, et al. Vital signs: cervical cancer incidence, mortality, and screening-United States, 2007-2012. MMWR Morb Mortal Wkly Rep 2014;63:1004-1009.

36. Brown ML, Klabunde CN, Cronin KA, White MC, Richardson LC, McNeel TS. Challenges in meeting Healthy People 2020 objectives for cancer-related preventive services, National Health Interview
Survey, 2008 and 2010. Prev Chronic Dis 2014;11:E29.

37. Scarinci IC, Garcia FA, Kobetz E, Partridge EE, Brandt HM, Bell $\mathrm{MC}$, et al. Cervical cancer prevention: new tools and old barriers. Cancer 2010;116:2531-2542.

38. Franco EL, Cuzick J, Hildesheim A, de Sanjosé S. Chapter 20: issues in planning cervical cancer screening in the era of HPV vaccination. Vaccine 2006;24 Suppl 3:S3/171-S3/177. 